\title{
JUMPING NUMBERS OF HYPERPLANE ARRANGEMENTS
}

\author{
NERO BUDUR
}

\begin{abstract}
M. Saito $[\underline{S}$ ] proved that the jumping numbers of a hyperplane arrangement depend only on the combinatorics of the arrangement. However, a formula in terms of the combinatorial data was still missing. In this note, we give a formula and a different proof of the fact that the jumping numbers of a hyperplane arrangement depend only on the combinatorics. We also give a combinatorial formula for part of the Hodge spectrum and for the inner jumping multiplicities.
\end{abstract}

\section{INTRODUCTION}

Jumping numbers are numerical measures of the complexity of the singularities of a variety (see section 22). M. Saito [S] proved that the jumping numbers of a reduced hyperplane arrangement depend only on the combinatorics of the arrangement, answering a question of M. Mustaţă [M]. The method of his proof was by reduction to the corresponding statement about the Hodge spectrum. His proof extends to non-reduced arrangements as well by taking into account the multiplicities along the hyperplanes in the arrangement. However, a formula in terms of the combinatorial data was still missing.

In this note, we give a formula and a different proof of the fact that the jumping numbers of a hyperplane arrangement depend only on the combinatorics and the multiplicities along hyperplanes. We also give a combinatorial formula for part of the Hodge spectrum and for the inner jumping multiplicities. Combinatorial formulas for those jumping numbers which change the support of the multiplier ideals have been obtained in [M]- Example 2.3, for reduced arrangements, and refined by $[\mathrm{T}]$ - Remark 3.2 .

Let $\mathcal{A}$ be a central hyperplane arrangement in $\mathbb{C}^{n}$. Denote the intersection lattice of $\mathcal{A}$ by $L(\mathcal{A})$, that is the set of subspaces of $\mathbb{C}^{n}$ which are intersections of subspaces $V \in \mathcal{A}$. We consider the corresponding arrangement of projective hyperplanes in $Y=\mathbb{P}^{n-1}$ given by $\mathbb{P}(V)$ for $V \in \mathcal{A}$. Let $D$ be an effective divisor on $Y$ supported on $\operatorname{Supp}(D)=\cup_{V \in \mathcal{A}} \mathbb{P}(V)$. We assume that $\operatorname{Supp}(D)$ is the compactification of a central hyperplane arrangement in some $\mathbb{C}^{n-1} \subset Y$. For our purposes, the general case can be reduced to this particular case.

We will give a combinatorial criterion, in terms of $L(\mathcal{A})$ and the multiplicities of $D$, for a positive rational number to be a jumping number of $D$ in $Y$. It is known

Date: February 13, 2008.

2000 Mathematics Subject Classification. 32S22.

Key words and phrases. arrangements, multiplier ideals, spectrum.

The author was supported by the NSF grant DMS-0700360. 
that 1 is trivially a jumping number of $D$ and that $c>1$ is a jumping number if and only if $c-1$ is. Thus it is enough to determine which $c \in(0,1)$ are jumping numbers of $D$.

Let $\mathcal{G}^{\prime} \subset L(\mathcal{A})-\left\{\mathbb{C}^{n}\right\}$ be a building set (see [DP]-2.4 or [T]-Definition 1.2). Let $\mathcal{G}=\mathcal{G}^{\prime} \cup\{0\}$. For simplicity, one can stick with the following example for the rest of the article: $\mathcal{G}=L(\mathcal{A}) \cup\{0\}-\left\{\mathbb{C}^{n}\right\}$, when $\mathcal{G}^{\prime}$ is chosen to be $L(\mathcal{A})-\left\{\mathbb{C}^{n}\right\}$. The advantage of considering smaller building sets is that computations might be faster (see [T]-Example 1.3-(c)).

For $V \in \mathcal{G}$, define $r(V)=\operatorname{codim}(V), \delta(V)=\operatorname{dim} V$, and

$$
s(V)=\sum_{V \subset W \in \mathcal{A}} \operatorname{mult}_{\mathbb{P}(W)}(D) .
$$

Set $d=\sum_{V \in \mathcal{A}} \operatorname{mult}_{\mathbb{P}(V)}(D)$ and

$$
a_{0}=\max \left\{d-n-1, \sum_{W \in \mathcal{G}-\{0\}} \max \{0, s(W)-r(W)\}\right\} .
$$

For any finite set $\mathcal{S}$, set $|\mathcal{S}|$ to be the number of elements of $\mathcal{S}$. For a rational number $c$ let

$$
\mathcal{S}_{c}=\{V \in \mathcal{G}-\{0\} \mid \operatorname{cs}(V) \in \mathbb{Z}\}
$$

For $V \in \mathcal{G}$ let

$$
a_{V}(c)= \begin{cases}r(V)-1-\llcorner c s(V)\lrcorner & \text { if } V \in \mathcal{G}-\{0\}, \notin \mathcal{S}_{c}, \\ r(V)-c s(V) & \text { if } V \in \mathcal{G}-\{0\}, \in \mathcal{S}_{c}, \\ -a_{0} & \text { if } V=0\end{cases}
$$

For a nested subset $\mathcal{S}$ of $\mathcal{G}-\{0\}$ and for $V \in \mathcal{S} \cup\left\{\mathbb{C}^{n}\right\}$, denote by $V_{\mathcal{S}}$ the subspace $\sum W$ where the sum is over $W \varsubsetneqq V$ such that $W \in \mathcal{S}$. In other words, $V_{\mathcal{S}}$ is the maximal element of $\mathcal{S}$ which is $\varsubsetneqq V$. Set $V_{\mathcal{S}}=0$ if there is not such maximal element. Let $Q(x)=x /(1-\exp (-x))$ considered as an element of the formal power series ring $\mathbb{Q}[[x]]$.

Definition 1.1. Let $\mathcal{S}$ be a nested subset of $\mathcal{G}-\{0\}$ and let $V \in \mathcal{S} \cup\left\{\mathbb{C}^{n}\right\}$. For $W \in \mathcal{G}$ with $V_{\mathcal{S}} \subset W \varsubsetneqq V$ define a formal power series $P_{W}^{\mathcal{S}, V} \in \mathbb{Q}\left[\left[c_{W^{\prime}}\right]\right]_{W^{\prime} \in \mathcal{G}}$ as follows. If $W=V_{\mathcal{S}}$ set

$$
P_{W}^{\mathcal{S}, V}=Q\left(-\sum_{\substack{W^{\prime} \subset V_{\mathcal{S}} \\\left\{W^{\prime}\right\} \cup \mathcal{S} \subset \mathcal{G} \text { nested }}} c_{W^{\prime}}\right)^{\delta(V)-\delta\left(V_{\mathcal{S}}\right)} .
$$

If $W \neq V_{\mathcal{S}}$ define

$$
\begin{gathered}
P_{W}^{\mathcal{S}, V}=Q\left(-\sum_{\substack{W^{\prime} \nsubseteq W \\
\left\{W^{\prime}\right\} \cup \mathcal{S} \subset \mathcal{G} \text { nested }}} c_{W^{\prime}}\right)^{-(\delta(V)-\delta(W))} \cdot Q\left(c_{W}\right) \cdot \\
\cdot Q\left(-\sum_{\substack{W^{\prime} \subset W \\
\left\{W^{\prime}\right\} \cup \mathcal{S} \subset \mathcal{G} \text { nested }}} c_{W^{\prime}}\right)^{\delta(V)-\delta(W)} .
\end{gathered}
$$


Definition 1.2. Let $\mathcal{S}$ be a nested subset of $\mathcal{G}-\{0\}$. Let $0 \leq j \leq n-1-|\mathcal{S}|$. Define the polynomial $T_{j}^{\mathcal{S}} \in \mathbb{Q}\left[c_{V}\right]_{V \in \mathcal{G}}$ to be the homogeneous part of degree $j$ of the formal power series

$$
T^{\mathcal{S}}:=\prod_{V \in \mathcal{S} \cup\left\{\mathbb{C}^{n}\right\}} \prod_{\substack{V_{\mathcal{S}} \subset W \varsubsetneqq V \\ W \in \mathcal{G}}} P_{W}^{\mathcal{S}, V} .
$$

Let $I \subset \mathbb{Z}\left[c_{V}\right]_{V \in \mathcal{G}}$ be the ideal of $[\mathrm{DP}]-5.2$ (for the projective case, see Remark 4.3). Recall that $I$ depends only on $\mathcal{G}$ and that $\mathbb{Z}\left[c_{V}\right]_{V \in \mathcal{G}} / I$ is isomorphic to the cohomology ring of the canonical log resolution in terms of $\mathcal{G}$ of $(Y, D)$, i.e. the wonderful model of [DP]. More precisely, $I$ is generated by two types of polynomials:

$$
\prod_{V \in \mathcal{H}} c_{V}
$$

if $\mathcal{H} \subset \mathcal{G}$ is not a nested subset, and by

$$
\prod_{V \in \mathcal{H}} c_{V}\left(\sum_{W^{\prime} \subset W} c_{W^{\prime}}\right)^{d_{\mathcal{H}, W}}
$$

where $\mathcal{H} \subset \mathcal{G}$ is a nested subset, $W \in \mathcal{G}$ is such that $W \varsubsetneqq V$ for all $V \in \mathcal{H}$, and $d_{\mathcal{H}, W}=\delta\left(\cap_{V \in \mathcal{H}} V\right)-\delta(W)$. In (2), one considers $\mathcal{H}=\emptyset$ to be nested, in which case (2) is defined for every $W \in \mathcal{G}$ by setting $\delta(\emptyset)=n$.

Theorem 1.3. With the notation as above, a rational number $c \in(0,1)$ is a jumping number of $D \subset Y$ if and only if

$$
\sum_{\substack{n e s t e d \\ \mathcal{S} \subset \mathcal{S}_{c}}} \sum_{0 \leq j}^{n-1-|\mathcal{S}|} \frac{(-1)^{|\mathcal{S}|+1}}{j !}\left(\sum_{V \in \mathcal{G}} a_{V}(c) c_{V}\right)^{j} T_{n-1-|\mathcal{S}|-j}^{\mathcal{S}} \prod_{V \in \mathcal{S}} c_{V}
$$

does not belong to the ideal $I \subset \mathbb{Q}\left[c_{V}\right]_{V \in \mathcal{G}}$.

Since we are assuming that $D$ is the compactification of a central hyperplane arrangement in $\mathbb{C}^{n-1}$, let $x \in Y$ be the point corresponding to the origin of $\mathbb{C}^{n-1}$. As for jumping numbers, the method of the proof of Theorem 1.3 gives a formula in terms of combinatorics for the inner jumping multiplicities $n_{c, x}(D)$ of a positive rational number $c$ along $D$ at the point $x$ (see section 2).

Theorem 1.4. With the notation as above, let $c$ be a positive rational number. Then the inner jumping multiplicity of $c$ along $D$ at $x$ is 0 if there are no subspaces $V \in \mathcal{G}$ with $\delta(V)=1$ or if $\mathrm{cm}(D) \notin \mathbb{Z}$. Otherwise, let $V_{x} \in \mathcal{G}$ be the only subspace with $\delta=1$, that is $\mathbb{P}\left(V_{x}\right)=\{x\}$. Then

$$
n_{c, x}(D)=\sum_{0 \leq j \leq n-2} \frac{1}{j !}\left(\sum_{V \in \mathcal{G}-\{0\}} a_{V}(c) c_{V}\right)^{j} T_{n-2-j}^{\left\{V_{x}\right\}} c_{V_{x}},
$$

where the right-hand side is viewed as a number via identification of the degree $n-1$ homogeneous part of $\mathbb{Q}\left[c_{V}\right]_{V \in \mathcal{G}} / I$ with $\mathbb{Q} \cdot\left(-c_{0}\right)^{n-1}$. 
By a result of [B] (see also [BS]), for $c \in(0,1]$ the inner jumping multiplicities $n_{c, x}(D)$ are the multiplicities of $c$ in the Hodge spectrum of $D$ at $x$ ([St]). Thus we have a combinatorial formula for the beginning part of the Hodge spectrum of a central hyperplane arrangement.

In section 2 we review multiplier ideals and intersection theory. In section 3 we set the problem into global setting, in preparation for using the Hirzebruch-RiemannRoch theorem. In section 4, we prove Theorems 1.3 and 1.4 via HirzebruchRiemann-Roch on wonderful models. In the last section we give an example illustrating how Theorems 1.3 and 1.4 work.

In this article, inclusion of sets is denoted by $\subset$ and strict inclusion of sets is denoted by $\subsetneq$.

We would like to thank M. Saito, to whom we are indebted for the proof of Lemma 3.2, for sharing with us the results of his preprint [S] which was the inspiration behind this article, and for many comments. We also thank M. Mustaţă and Z. Teitler for useful discussions. The author was supported by the NSF grant DMS0700360 .

\section{REVIEW OF MULTIPLIER IDEALS, INTERSECTION THEORY}

The notation of the current section is independent of the rest of the article.

Multiplier ideals. We review some basic facts from the theory of multiplier ideals (see [L-Chapter 9). Let $Y$ be a smooth complex variety. Let $D$ be an effective $\mathbb{Q}$-divisor on $Y$. Let $\rho: Y^{\prime} \rightarrow Y$ be a $\log$ resolution of $(Y, D)$ and let $K_{Y^{\prime} / Y}$ be the relative canonical divisor. The multiplier ideal of $D$ is the ideal sheaf

$$
\mathcal{J}(D)=\rho_{*} \mathcal{O}_{Y^{\prime}}\left(K_{Y^{\prime} / Y}-\left\llcorner\rho^{*} D\right\lrcorner\right) \quad \subset \mathcal{O}_{Y}
$$

The choice of log resolution does not matter in the definition of the $\mathcal{J}(D)$ and one can extend the definition by allowing, instead of $D$, any finite formal linear combination of subschemes of $Y$ with positive coefficients. A positive rational number $c$ is called a jumping number of $D$ if $\mathcal{J}(c \cdot D) \neq \mathcal{J}((c-\epsilon) \cdot D)$ for all $0<\epsilon \ll 1$. It is known that a positive rational number $c$ is a jumping number if and only if $c+1$ is a jumping number ([L]- Example 9.3.24). Let $x$ be a point in the support of $D$ and let $c>0$. The inner jumping multiplicity of $c$ along $D$ at $x$ ([B]- Definition 2.4) is defined as

$$
n_{c, x}(D)=\operatorname{dim}_{\mathbb{C}} \frac{\mathcal{J}((c-\epsilon) D)}{\mathcal{J}((c-\epsilon) D+\delta\{x\})},
$$

where $0<\epsilon \ll \delta \ll 1$. By $[\mathrm{B}]$-Proposition 2.8, if the inner jumping multiplicity of $c$ is nonzero then $c$ is a jumping number.

Theorem 2.1. (Local vanishing, $[\mathrm{L}]$ - Theorem 9.4.1). With the notation as above,

$$
R^{j} \rho_{*} \mathcal{O}_{Y^{\prime}}\left(K_{Y^{\prime} / Y}-\left\llcorner\rho^{*} D\right\lrcorner\right)=0 \quad \text { for } j>0 .
$$


Theorem 2.2. (Nadel vanishing, $[\mathrm{L}$ - Theorem 9.4.9). With the notation as above, assume in addition that $Y$ is projective. Let $L$ be any integral divisor such that $L-D$ is nef and big. Then

$$
H^{i}\left(Y, \mathcal{O}_{Y}\left(K_{Y}+L\right) \otimes \mathcal{J}(D)\right)=0 \quad \text { for } i>0
$$

Intersection theory. We recall some facts about intersection theory (see $[\mathrm{F}]$ ). Let $Y$ be a smooth projective complex variety. For a vector bundle, or locally free $\mathcal{O}_{Y}$-module of finite $\operatorname{rank}, \mathcal{E}$ on $Y$, we denote by $c_{j}(\mathcal{E})$ the image of the $j$ th Chern class of $\mathcal{E}$ in $H^{2 j}(Y, \mathbb{Z})$. The total Chern class is defined to be $c(\mathcal{E})=$ $\sum_{j} c_{j}(\mathcal{E})$ in the cohomology $\operatorname{ring} H^{*}(Y, \mathbb{Z})$. The roots $x_{i}$ of $\mathcal{E}$ are formal symbols satisfying the formal decomposition $\sum_{j} c_{j}(\mathcal{E}) t^{j}=\prod_{i}\left(1+x_{i} t\right)$. Then one defines $\operatorname{ch}(\mathcal{E})=\sum_{i} \exp \left(x_{i}\right)$, and writes $c h(\mathcal{E})=\sum_{j} c h_{j}(\mathcal{E})$ with $c h_{j}(\mathcal{E}) \in H^{2 j}(Y, \mathbb{Q})$. The Todd class of $\mathcal{E}$ is defined as $t d(\mathcal{E})=\prod Q\left(x_{i}\right)$, where $Q(x)=x /(1-\exp (-x))$. The Todd class of $Y$ is denoted by $\operatorname{Td}(Y)$ and is defined as the Todd class of the tangent bundle of $Y$. One writes $T d(Y)=\sum_{j} T d_{j}(Y)$ where $T d_{j}(Y) \in H^{2 j}(Y, \mathbb{Q})$.

Theorem 2.3. (Hirzebruch-Riemann-Roch, [F]-Corollary 15.2.1) Let $\mathcal{E}$ be a vector bundle on a smooth projective complex variety $Y$. Then $\chi(Y, \mathcal{E})$ is the intersection number $\sum_{i+j=\operatorname{dim} Y} \operatorname{ch}_{i}(\mathcal{E}) \cdot T d_{j}(Y)$.

Let $X_{1}, \ldots X_{t}$ be disjoint smooth subvarieties of $Y$ of codimension $d$. Let $\rho: \widetilde{Y} \rightarrow$ $Y$ be the blow up of $\coprod_{\widetilde{Y}} X_{i}$. Let $E_{i}$ be the exceptional divisor on $\widetilde{Y}$ corresponding to $X_{i}$. Let $\left[E_{i}\right] \in H^{2}(\widetilde{Y}, \mathbb{Z})$ be the cohomology class of $E_{i}$. Let $N_{i}$ be the normal bundle of $X_{i}$ in $Y$. Suppose there exist $c_{k, i} \in H^{2 k}(Y, \mathbb{Z})$ such that the Chern classes $c_{k}\left(N_{i}\right)$ are the restriction of $c_{k, i}$ to $X_{i}$. The following computes the total Chern class of $\widetilde{Y}$ and follows from [F]-Example 15.4.2.

Proposition 2.4. With the notation as above,

$$
c(\tilde{Y})=\rho^{*} c(Y) \prod_{1 \leq j \leq t}\left\{\left(\sum_{0 \leq k \leq d} \rho^{*} c_{k, j}\right)^{-1}\left(1+\left[E_{j}\right]\right)\left(\sum_{0 \leq i \leq d}\left(1-\left[E_{j}\right]\right)^{i} \rho^{*} c_{d-i, j}\right)\right\} .
$$

\section{UNIFORM BOUND FOR JUMPS IN MULTIPLIER IDEALS}

Affine case. Let $\mathcal{A}^{\prime}$ be a central hyperplane arrangement in $\mathbb{C}^{n-1}$. Let $D^{\prime}$ be an effective divisor on $\mathbb{C}^{n-1}$ with support $\mathcal{A}^{\prime}$. Let $L\left(\mathcal{A}^{\prime}\right)$ be the intersection lattice of $\mathcal{A}^{\prime}$. For $V \in L\left(\mathcal{A}^{\prime}\right)$, define $r^{\prime}(V)=\operatorname{codim}(V)$ and $s^{\prime}(V)=\sum_{V \subset W \in \mathcal{A}^{\prime}} \operatorname{mult}_{W}\left(D^{\prime}\right)$. Let $\mathcal{G}^{\prime} \subset L\left(\mathcal{A}^{\prime}\right)-\left\{\mathbb{C}^{n-1}\right\}$ be a building set. Recall the following result of $\mathrm{M}$. Mustaţă [M]-Corollary 2.1 for the case of reduced arrangements, and refined by Teitler [T]-Theorem 1.4.

Proposition 3.1. If $D^{\prime}$ is an effective divisor supported on a central hyperplane arrangement in $\mathbb{C}^{n-1}$, then

$$
\mathcal{J}\left(c D^{\prime}\right)=\bigcap_{W \in \mathcal{G}^{\prime}} I_{W}^{\left\ulcorner c s^{\prime}(W)\right\urcorner-r^{\prime}(W)} .
$$


Moreover, $c$ is a jumping number of $D^{\prime}$ if and only if there are $V \in \mathcal{G}^{\prime}$ and $m \in \mathbb{N}$ such that $c=\frac{r^{\prime}(V)+m}{s^{\prime}(V)}$ and such that

$$
\bigcap_{V \subset W \in \mathcal{G}^{\prime}} I_{W}^{\left\ulcorner c s^{\prime}(W)\right\urcorner-r^{\prime}(W)} \not \subset I_{V}^{m+1} .
$$

The following lemma will allow us to bound the degrees of the polynomials at which we need to look to detect a jump of multiplier ideals. We have conjectured the statement, proved some cases, and M. Saito proved it in general.

Lemma 3.2. For $1 \leq i \leq s$, let $I_{i} \subset \mathbf{C}\left[x_{1}, \ldots, x_{n}\right]$ be ideals generated by linear forms. Suppose $I_{1}^{a_{1}} \cap \ldots \cap I_{s}^{a_{s}} \not \subset I_{1}^{a_{1}+1}$ for some positive integers $a_{i}$. Then there exists $f$ in $I_{1}^{a_{1}} \cap \ldots \cap I_{s}^{a_{s}}$ but not in $I_{1}^{a_{1}+1}$ of degree at most $a_{1}+\ldots+a_{s}$.

Proof. The following short and elementary proof of this lemma is due M. Saito who kindly allowed us to reproduce it here. After a change of coordinates, we can assume that $I_{1}=\left(x_{1}, \ldots, x_{m}\right)$ for some $m \leq n$. After reordering of indices, we can assume that there is $r \in\{1, \ldots, s\}$ such that $I_{i} \subset I_{1}$ for $1 \leq i \leq r$ and $I_{i} \not \subset I_{1}$ for $r<i \leq s$. Let $J_{i}=I_{i} \cap \mathbb{C}\left[x_{1}, \ldots, x_{m}\right]$. Then

$$
\bigcap_{1 \leq i \leq r} I_{i}^{a_{i}}=\bigcap_{1 \leq i \leq r} J_{i}^{a_{i}} \cdot \mathbb{C}\left[x_{1}, \ldots, x_{n}\right] .
$$

Since $\cap_{1 \leq i \leq r} I_{i}^{a_{i}} \not \subset I_{1}^{a_{1}+1}$, we have that $\cap_{1 \leq i \leq r} J_{i}^{a_{i}} \not \subset J_{1}^{a_{1}+1}$. The ideals $J_{i}$ are homogeneous. Hence we can find a homogeneous polynomial $u$ in $\cap_{1 \leq i \leq r} J_{i}^{a_{i}}$ which does not belong to $J_{1}^{a_{1}+1}=\left(x_{1}, \ldots, x_{m}\right)^{a_{1}+1}$. Then the degree of $u$ must be $a_{1}$. For $r<i \leq s$, take $v_{i} \in I_{i}$ but $\notin I_{1}$ to be a linear form. Let $f=u \prod_{r<i \leq s} v_{i}^{a_{i}}$. Then $f \in \cap_{1 \leq i \leq s} I_{i}^{a_{i}}$, but $f \notin I_{1}^{a_{1}+1}$, and the degree of $f$ is $a_{1}+a_{r+1}+\ldots+a_{s}$.

Let $a_{0}^{\prime}=\sum_{W \subset \mathcal{G}^{\prime}} \max \left\{0, s^{\prime}(W)-r^{\prime}(W)\right\}$. By Proposition 3.1 and Lemma 3.2, we have:

Corollary 3.3. If $D^{\prime}$ is an effective divisor supported on a central hyperplane arrangement in $\mathbb{C}^{n-1}$, then $c \in(0,1)$ is a jumping number of $D^{\prime}$ if and only if there exists $f \in \mathbb{C}\left[x_{1}, \ldots, x_{n-1}\right]$ of degree at most $a_{0}^{\prime}$ with $f \in \mathcal{J}\left((c-\epsilon) D^{\prime}\right)$ for $0<\epsilon \ll 1$, but $f \notin \mathcal{J}\left(c D^{\prime}\right)$.

Projective case. Let $\mathcal{A}$ be a central hyperplane arrangement in $\mathbb{C}^{n}$. Denote the intersection lattice of $\mathcal{A}$ by $L(\mathcal{A})$. We consider the corresponding arrangement of projective hyperplanes in $Y=\mathbb{P}^{n-1}$ given by $\mathbb{P}(V)$ for $V \in \mathcal{A}$. Let $D$ be an effective divisor on $Y$ supported on $\cup_{V \in \mathcal{A}} \mathbb{P}(V)$. Assume that the support of $D$ is the compactification of a central hyperplane arrangement in some $\mathbb{C}^{n-1} \subset Y$. Let $\mathcal{G}^{\prime} \subset$ $L(\mathcal{A})-\left\{\mathbb{C}^{n}\right\}$ be a building set and let $\mathcal{G}=\mathcal{G}^{\prime} \cup\{0\}$. For $c$ a positive real number, let $\mathcal{J}(c D)$ be the multiplier ideal of $c D$ in $Y$. Let $\mathcal{G}(c D)=\mathcal{J}((c-\epsilon) D) / \mathcal{J}(c D)$ for $0<\epsilon \ll 1$. Thus $c$ is a jumping number of $D$ if and only if $\mathcal{G}(c D) \neq 0$. Recall that we defined in the introduction, for $V \in \mathcal{G}-\{0\}$, the numbers $r(V)$ and $s(V)$. Let $a_{0}$ be defined as in the introduction. By Corollary [3.3, we have: 
Corollary 3.4. For all $c \in(0,1)$,

$$
\mathcal{G}(c D) \neq 0 \Leftrightarrow H^{0}\left(Y, \mathcal{O}_{Y}\left(a_{0}\right) \otimes \mathcal{G}(c D)\right) \neq 0 .
$$

\section{INTERSECTION THEORY ON CANONICAL LOG RESOLUTIONS.}

The canonical $\log$ resolution. Let $\mathcal{A}$ be a central hyperplane arrangement in $\mathbb{C}^{n}$. We consider the corresponding arrangement of projective hyperplanes in $Y=\mathbb{P}^{n-1}$ given by $\mathbb{P}(V)$ for $V \in \mathcal{A}$. Let $D$ be an effective divisor on $Y$ supported on $\cup_{V \in \mathcal{A}} \mathbb{P}(V)$. We assume also that the support of $D$ is the compactification of a central hyperplane arrangement in some $\mathbb{C}^{n-1} \subset Y$. Let $\mathcal{G}^{\prime} \subset L(\mathcal{A})-\left\{\mathbb{C}^{n}\right\}$ be a building set. Let $\mathcal{G}=\mathcal{G}^{\prime} \cup\{0\}$. For example, $\mathcal{G}=L(\mathcal{A}) \cup\{0\}-\left\{\mathbb{C}^{n}\right\}$.

We consider the canonical log resolution $\rho: \widetilde{Y} \rightarrow Y$ of $D$ obtained from succesive blowing ups of the (disjoint) unions of (the proper transforms) of $\mathbb{P}(V)$ for $V \in$ $\mathcal{G}-\{0\}$ of same dimension. This is the so-called wonderful model of [DP]- section 4. More precisely, $\rho$ and $\widetilde{Y}$ are constructed as follows.

The following notation is taken from $[\mathrm{S}]$ - section 2. Let $Y_{0}=Y$. Let $C_{0}$ be $\mathbb{P}(V)$ for $V \in \mathcal{G}-\{0\}$ with $\delta(V)=1$ (there is at most one such $V$, by assumption). Let $\rho_{0}: Y_{1} \rightarrow Y_{0}$ be the blow up of $C_{0}$. Then $\rho_{i}$ and $Y_{i+1}$ are constructed inductively as follows. Let $C_{i} \subset Y_{i}$ be the disjoint union of the proper transforms, under the map $\rho_{i-1}$, of $\mathbb{P}(V)$ for $V \in \mathcal{G}-\{0\}$ with $\delta(V)=i+1$. Let $\rho_{i}: Y_{i+1} \rightarrow Y_{i}$ for $0 \leq i<n-2$ be the blow up of $C_{i}$. Define $\widetilde{Y}=Y_{n-2}$ and $\rho$ as the composition of the $\rho_{i}$.

We need some more notation, also from [S]- section 2. Let $C_{V, 0}=\mathbb{P}(V) \subset Y_{0}$. For $V \in \mathcal{G}-\{0\}$ with $\delta(V)=i+1, C_{V, j}$ denotes the proper transform of $C_{V, 0}$ in $Y_{j}$ for $1 \leq j \leq i$. Let $E_{V, i+1}$ be the exceptional divisor in $Y_{i+1}$ corresponding to $C_{V, i}$. Let $E_{V, j}$ be the proper transform of $E_{V, i+1}$ in $Y_{j}$ for $i+1<j \leq n-2$. On $\widetilde{Y}$, let $E_{V}=E_{V, n-2}$ if $\delta(V)<n-1$, and $E_{V}=C_{V, n-2}$ if $\delta(V)=n-1$. Also let $E_{0, i}$ $(0 \leq i \leq n-2)$, and $E_{0}$, denote the proper transform in $Y_{i}$, respectively in $\tilde{Y}$, of a general hyperplane of $Y=\mathbb{P}^{n-1}$. Denote by $\left[E_{V}\right]$ the cohomology class of $E_{V}$ on $\widetilde{Y}$, where it will be clear from context what coefficients (integral, rational) we are considering.

For any subset $\mathcal{S}$ of $\mathcal{G}-\{0\}$, set $E^{\mathcal{S}}=\cup_{V \in \mathcal{S}} E_{V}$ and $E_{\mathcal{S}}=\cap_{V \in \mathcal{S}} E_{V}$. For a rational number $c$, recall the definitions of $\mathcal{S}_{c}, a_{0}$, and $a_{V}(c)$ from the introduction. Also define $a_{V}^{\prime}(c)$ to equal $a_{V}(c)$ for $c \neq 0$ and, otherwise, $a_{V}^{\prime}(0)=a_{0}$.

Lemma 4.1. With the notation as above,

$$
H^{0}\left(Y, \mathcal{O}_{Y}\left(a_{0}\right) \otimes \mathcal{G}(c D)\right)=\chi\left(\mathcal{O}_{E^{\mathcal{S}_{c}}}\left(\sum_{V \in \mathcal{G}} a_{V}^{\prime}(c) E_{V}\right)\right) .
$$

Proof. We have that $K_{\widetilde{Y} / Y}=\sum_{V \in \mathcal{G}-\{0\}}(r(V)-1) E_{V}$ and $\rho^{*}(D)=\sum_{V \in \mathcal{G}-\{0\}} s(V) E_{V}$ ([T]-Lemma 2.1). Then, from the definition of multiplier ideals and Theorem 2.1, 
we have

$$
\begin{aligned}
\mathcal{G}(c D) & =\rho_{*}\left(\mathcal{O}_{E} \mathcal{S}_{c}\left(\sum_{V \in \mathcal{G}-\{0\}} a_{V}(c) E_{V}\right)\right), \text { and } \\
0 & =R^{i} \rho_{*}\left(\mathcal{O}_{E^{\mathcal{S}_{c}}}\left(\sum_{V \in \mathcal{G}-\{0\}} a_{V}(c) E_{V}\right)\right) \text { for } i>0 .
\end{aligned}
$$

We can rewrite $\mathcal{O}_{Y}\left(a_{0}\right)$ as $\omega_{Y} \otimes \mathcal{O}_{Y}\left(a_{0}+n+2\right)$. By definition, $a_{0}+n+2>m(D)$. Hence Theorem 2.2 applies and we have

$$
\begin{aligned}
H^{0}\left(Y, \mathcal{O}_{Y}\left(a_{0}\right) \otimes \mathcal{G}(c D)\right) & =\chi\left(\mathcal{O}_{\widetilde{Y}}\left(a_{0} E_{0}\right) \otimes \mathcal{O}_{E \mathcal{S}_{c}}\left(\sum_{V \in \mathcal{G}-\{0\}} a_{V}(c) E_{V}\right)\right) \\
& =\chi\left(\mathcal{O}_{E^{\mathcal{S}_{c}}}\left(\sum_{V \in \mathcal{G}} a_{V}^{\prime}(c) E_{V}\right)\right) .
\end{aligned}
$$

Lemma 4.2. With the notation as in Lemma 4.1, a rational number $c \in(0,1)$ is a jumping number of $D$ if and only if

$$
\sum_{\text {nested } \mathcal{S} \subset \mathcal{S}_{c}}(-1)^{|\mathcal{S}|+1} \chi\left(\mathcal{O}_{E_{\mathcal{S}}}\left(\sum_{V \in \mathcal{G}} a_{V}^{\prime}(c) E_{V}\right)\right) \neq 0 .
$$

Proof. Follows from Lemma 4.1 and Corollary 3.4 via the Mayer-Vietoris exact sequence

$$
0 \rightarrow \mathcal{O}_{E^{\mathcal{S}_{c}}} \rightarrow \bigoplus_{\substack{\mathcal{S} \subset \mathcal{S}_{c} \\|\mathcal{S}|=1}} \mathcal{O}_{E_{\mathcal{S}}} \rightarrow \bigoplus_{\substack{\mathcal{S} \subset \mathcal{S}_{c} \\|\mathcal{S}|=2}} \mathcal{O}_{E_{\mathcal{S}}} \rightarrow \ldots \rightarrow \mathcal{O}_{E_{\mathcal{S}_{c}}} \rightarrow 0
$$

The intersection $E_{\mathcal{S}}$ is nonempty if and only if $\mathcal{S}$ is nested ([S]-2.7, [DP]-4.2).

Next goal is to compute $\chi\left(\mathcal{O}_{E_{\mathcal{S}}}\left(\sum_{V \in \mathcal{G}} a_{V}^{\prime}(c) E_{V}\right)\right)$ via Hirzebruch-RiemannRoch.

Remark 4.3. Let $I \subset \mathbb{Z}\left[c_{V}\right]_{V \in \mathcal{G}}$ be the ideal of $[\mathrm{DP}]-5.2$ described in the introduction. By loc. cit. there is an isomorphism

$$
\begin{aligned}
& \mathbb{Z}\left[c_{V}\right]_{V \in \mathcal{G}} / I \stackrel{\sim}{\longrightarrow} H^{*}(\tilde{Y}, \mathbb{Z}) \stackrel{\sim}{\longleftarrow}\left[\left[c_{V}\right]\right]_{V \in \mathcal{G}} / I \\
& c_{V} \mapsto\left[E_{V}\right] \quad \text { if } V \neq 0 \text {, } \\
& c_{0} \mapsto-\left[E_{0}\right] .
\end{aligned}
$$

Indeed, this follows from [DP]-5.2 Theorem, [DP]-4.1 Theorem, part (2), and [DP]4.2 Theorem, part (4). The only case left out by [DP]-4.2 Theorem, part (4) is the one corresponding with $E_{0}$ in our notation. But this follows from the fact that, in their notation, the linear equivalence class of $D_{V^{*}}$ restricted to $D_{V^{*}}$ is the negative of the class of the proper transform in $D_{V^{*}}$ of a general hyperplane in the exceptional divisor of the blowup of the origin of $V$. The objects $V$ and $D_{V^{*}}$ of [DP] correspond to $\mathbb{C}^{n}$ and, respectively, $\widetilde{Y}$, in our notation. The exceptional 
divisor of the blowup of the origin of $V$ is, in our notation, $\mathbb{P}^{n-1}$, the ambient space of our projective arrangement of hyperplanes.

Lemma 4.4. With the notation as in Theorem 1.3, let $\mathcal{S}$ be a nested subset of $\mathcal{G}-\{0\}$, and $c$ a rational number. Then

$$
\begin{aligned}
& \chi\left(\mathcal{O}_{E_{\mathcal{S}}}\left(\sum_{V \in \mathcal{G}} a_{V}^{\prime}(c) E_{V}\right)\right)= \\
= & \sum_{0 \leq j}^{n-1-|\mathcal{S}|} \frac{1}{(n-1-|\mathcal{S}|-j) !}\left(\sum_{V \in \mathcal{G}} a_{V}(c) c_{V}\right)^{n-1-|\mathcal{S}|-j} T_{j}^{\mathcal{S}} \prod_{V \in \mathcal{S}} c_{V},
\end{aligned}
$$

where the right-hand side is viewed as an intersection number via the isomorphism (3).

Proof of Theorem 1.3. It follows from Lemma 4.2 and Lemma 4.4.

Before we prove Lemma 4.4, we need some preliminary results.

Write $Y=\mathbb{P}\left(\mathbb{C}^{n}\right)$ and $\tilde{Y}=\mathbb{P}\left(\mathbb{C}^{n}\right)^{\mathcal{G}}$. This notation makes sense if one replaces $\mathbb{C}^{n}$ and $\mathcal{G}$ by any vector space with a finite set of proper vector subspaces which is closed under intersections and contains $\{0\}$. For a nested subset $\mathcal{S} \subset \mathcal{G}-\{0\}$ and $V \in \mathcal{S} \cup\left\{\mathbb{C}^{n}\right\}$, let $V_{\mathcal{S}}$ be as in introduction. Define $\mathbb{C}_{V}^{\mathcal{S}}=V / V_{\mathcal{S}}$ and set

$\mathcal{G}_{V}^{\mathcal{S}}=\left\{W^{\prime} \subset \mathbb{C}_{V}^{\mathcal{S}} \mid W^{\prime}\right.$ is the image of $W$ in $\mathbb{C}_{V}^{\mathcal{S}}$ for some $\left.W \in \mathcal{G}, W \subset V\right\}$.

We have the following description of $E_{\mathcal{S}}([\underline{\mathrm{S}}]-2.7$, [DP]-4.3):

Proposition 4.5. With the notation as above, let $\mathcal{S} \subset \mathcal{G}-\{0\}$ be a nested subset. Then

$$
E_{\mathcal{S}}=\prod_{V \in \mathcal{S} \cup\left\{\mathbb{C}^{n}\right\}} \mathbb{P}\left(\mathbb{C}_{V}^{\mathcal{S}}\right)^{\mathcal{G}_{V}^{\mathcal{S}}}
$$

By $[\mathrm{F}]$ - Example 15.2.12, the Todd class of $E_{\mathcal{S}}$ is also a product:

Lemma 4.6. With the notation as in Proposition 4.5 ,

$$
\operatorname{Td}\left(E_{\mathcal{S}}\right)=\prod_{V \in \mathcal{S} \cup\left\{\mathbb{C}^{n}\right\}} \operatorname{Td}\left(\mathbb{P}\left(\mathbb{C}_{V}^{\mathcal{S}}\right)^{\mathcal{G}_{V}^{\mathcal{S}}}\right) .
$$

More precisely, $\operatorname{Td}\left(E_{\mathcal{S}}\right)$ is the product of the pullbacks of $\operatorname{Td}\left(\mathbb{P}\left(\mathbb{C}_{V}^{\mathcal{S}}\right)^{\mathcal{G}_{V}^{\mathcal{S}}}\right)$ under the projections associated to the decomposition in Proposition 4.5 .

For every $V \in \mathcal{G}-\{0\}$ define a formal power series $F_{V} \in \mathbb{Z}\left[\left[c_{V}\right]\right]_{V \in \mathcal{G}}$ by

$$
F_{V}:=\left(1-\sum_{\substack{W \varsubsetneqq V \\ W \in \mathcal{G}}} c_{W}\right)^{-(n-\delta(V))}\left(1+c_{V}\right)\left(1-\sum_{\substack{W \subset V \\ W \in \mathcal{G}}} c_{W}\right)^{n-\delta(V)} .
$$

Also, set $F_{0}=\left(1-c_{0}\right)^{n}$.

Proposition 4.7. With the notation as above, the total Chern class $c(\widetilde{Y})$ is the image in $H^{*}(\widetilde{Y}, \mathbb{Z})$ of $\prod_{V \in \mathcal{G}} F_{V}$ under the map (3). 
Proof. For $V \in \mathcal{G}-\{0\}$ with $\delta(V)=i+1$, let $N_{V, i}$ be the normal bundle of $C_{V, i}$ in $Y_{i}$. Let

$$
\left.L_{V, i}=\mathcal{O}_{Y_{i}}\left(E_{0, i}-\sum_{\substack{0 \neq W \varsubsetneqq V \\ W \in \mathcal{G}}} E_{W, i}\right)\right) .
$$

By [DP]-5.1 (the statement in loc. cit. needs to be adjusted for the projective case as in Remark 4.3),

$$
N_{V, i} \cong L_{V, i}^{\oplus n-1-i} \mid C_{V, i} .
$$

We want to apply Proposition 2.4. One of the quantities we need is

$$
\begin{aligned}
{\left[\sum_{0 \leq k \leq n-1-i} \rho_{i}^{*} c_{k}\left(L_{V, i}{ }^{\oplus(n-1-i)}\right)\right]^{-1} } & =\rho_{i}^{*} c\left(L_{V, i}\right)^{-(n-1-i)} \\
& =\left(1+\left[E_{0, i+1}\right]-\sum_{\substack{0 \neq W \varsubsetneqq V \\
W \in \mathcal{G}}}\left[E_{W, i+1}\right]\right)^{-(n-1-i)} .
\end{aligned}
$$

Also, we have

$$
\begin{aligned}
& \sum_{0 \leq j \leq n-1-i}\left(1-\left[E_{V, i+1}\right]\right)^{j} \rho_{i}^{*} c_{n-1-i-j}\left(L_{V, i}^{\oplus}{ }^{n-1-i}\right)= \\
= & \sum_{0 \leq j \leq n-1-i}\left(1-\left[E_{V, i+1}\right]\right)^{j}\left(\begin{array}{c}
n-1-i \\
n-1-i-j
\end{array}\right) \rho_{i}^{*} c_{1}\left(L_{V, i}\right)^{n-1-i-j} \\
= & \left(1-\left[E_{V, i+1}\right]+\left[E_{0, i+1}\right]-\sum_{\substack{0 \neq W \varsubsetneqq V \\
W \in \mathcal{G}}}\left[E_{W, i+1}\right]\right)^{n-1-i}
\end{aligned}
$$

By Proposition 2.4

$$
c\left(Y_{i+1}\right)=\rho_{i}^{*} c\left(Y_{i}\right) \prod_{\substack{V \in \mathcal{G} \\
\delta(V)=i+1}}\left\{\begin{array}{c}
\left(1+\left[E_{0, i+1}\right]-\sum_{\substack{0 \neq W \varsubsetneqq V \\
W \in \mathcal{G}}}\left[E_{W, i+1}\right]\right)^{-(n-1-i)}\left(1+\left[E_{V, i+1}\right]\right) . \\
\left.\left(1-\left[E_{V, i+1}\right]+\left[E_{0, i+1}\right]-\sum_{\substack{0 \neq W \varsubsetneqq V \\
W \in \mathcal{G}}}\left[E_{W, i+1}\right]\right)^{n-1-i}\right\}
\end{array}\right.
$$

Since $\widetilde{Y}=Y_{n-2}$, the Proposition follows from the last formula.

Let $Q(x)=x /(1-\exp (-x))$. For every $V \in \mathcal{G}-\{0\}$ define a formal power series $G_{V}^{\mathcal{G}} \in \mathbb{Q}\left[\left[c_{V}\right]\right]_{V \in \mathcal{G}}$ by

$$
G_{V}^{\mathcal{G}}:=Q\left(-\sum_{\substack{W \nsubseteq V \\ W \in \mathcal{G}}} c_{W}\right)^{-r(V)} Q\left(c_{V}\right) Q\left(-\sum_{\substack{W \subset V \\ W \in \mathcal{G}}} c_{W}\right)^{r(V)} .
$$

Also, set $G_{0}^{\mathcal{G}}=Q\left(-c_{0}\right)^{n}=Q\left(-c_{0}\right)^{r(0)}$. Recall from introduction that the codimension function $r$ depends only $\mathcal{G}$, a fact which is suppressed from the notation. 
Since the Todd class, as the total Chern class, is multiplicative on exact sequences of vector bundles, by Proposition 4.7 we have:

Corollary 4.8. With the notation as in Proposition 4.7, the Todd class $\operatorname{Td}(\widetilde{Y})$ is the image in $H^{*}(\widetilde{Y}, \mathbb{Q})$ of $\prod_{V \in \mathcal{G}} G_{V}^{\mathcal{G}}$ under the map induced by (3) after $\otimes_{\mathbb{Z}} \mathbb{Q}$.

Replacing, in Corollary 4.8, $\widetilde{Y}=\mathbb{P}\left(\mathbb{C}^{n}\right)^{\mathcal{G}}$ with $\mathbb{P}\left(\mathbb{C}_{V}^{\mathcal{S}}\right)^{\mathcal{G}_{V}^{\mathcal{S}}}$, we obtain:

Corollary 4.9. With the notation as in Proposition 4.5 and Corollary 4.8, let $\mathcal{S} \subset \mathcal{G}-\{0\}$ be a nested subset and let $V \in \mathcal{S} \cup\left\{\mathbb{C}^{n}\right\}$. The Todd class $\operatorname{Td}\left(\mathbb{P}\left(\mathbb{C}_{V}^{\mathcal{S}}\right)^{\mathcal{G}_{V}^{\mathcal{S}}}\right)$ is the image in $H^{*}\left(\mathbb{P}\left(\mathbb{C}_{V}^{\mathcal{S}}\right)^{\mathcal{G}_{V}^{\mathcal{S}}}, \mathbb{Q}\right)$ of

$$
\prod_{W^{\prime} \in \mathcal{G}_{V}^{\mathcal{S}}} G_{W^{\prime}}^{\mathcal{G}_{V}^{\mathcal{S}}} \in \mathbb{Q}\left[\left[c_{W^{\prime \prime}}\right]\right]_{W^{\prime \prime} \in \mathcal{G}_{V}^{\mathcal{S}}}
$$

under the map $c_{W^{\prime \prime}} \mapsto\left[E_{W^{\prime \prime}}\right]\left(W^{\prime \prime} \neq 0\right)$ and $c_{0} \mapsto-\left[E_{0}\right]$.

Next lemma puts together some computations from [DP]-4.3, [S]-Propositions 2.8 and 2.9:

Lemma 4.10. With the notation as in Proposition 4.5, let $\mathcal{S} \subset \mathcal{G}-\{0\}$ be a nested subset. For $V \in \mathcal{S} \cup\left\{\mathbb{C}^{n}\right\}$, let $p_{V}$ be the projection of $E_{\mathcal{S}}$ onto the factor $\mathbb{P}\left(\mathbb{C}_{V}^{\mathcal{S}}\right)^{\mathcal{G}_{V}^{\mathcal{S}}}$ associated to the decomposition in Proposition 4.5. Let $W^{\prime} \in \mathcal{G}_{V}^{\mathcal{S}}$ with corresponding divisor $E_{W^{\prime}}^{\prime}$ in $\mathbb{P}\left(\mathbb{C}_{V}^{\mathcal{S}}\right)^{\mathcal{G}_{V}^{\mathcal{S}}}$.

(a) If $W^{\prime} \neq 0$, then $p_{V}^{*} E_{W^{\prime}}^{\prime} \sim E_{W \mid E_{\mathcal{S}}}$, where $W$ is the unique element of $\mathcal{G}$ nested between $V$ and $V_{\mathcal{S}}$ whose image in $\mathbb{C}_{V}^{\mathcal{S}}=V / V_{\mathcal{S}}$ is $W^{\prime}$.

(b) If $W^{\prime}=0$, then

$$
p_{V}^{*} E_{0}^{\prime} \sim\left(E_{0}-\sum_{\substack{0 \neq W \subset V_{\mathcal{S}}, W \in \mathcal{G} \\\{W\} \cup S \text { nested }}} E_{W}\right)_{\mid E_{\mathcal{S}}} .
$$

Proof. For $\mathcal{S}$ having only one element, this is [S]- Proposition 2.8. For the rest, one iterates as in [S]- Proposition 2.9 or, equivalently, as in the last paragraph of the proof of the theorem of $[\mathrm{DP}]-4.3$.

Proposition 4.11. With the notation as in Proposition 4.5 and Definition 1.1 , $\operatorname{Td}\left(E_{\mathcal{S}}\right)$ is the image of the formal power series

$$
T^{\mathcal{S}}:=\prod_{V \in \mathcal{S} \cup\left\{\mathbb{C}^{n}\right\}} \prod_{\substack{V_{\mathcal{S}} \subset W \varsubsetneqq V \\ W \in \mathcal{G}}} P_{W}^{\mathcal{S}, V} \in \mathbb{Q}\left[\left[c_{W}\right]\right]_{W \in \mathcal{G}}
$$

in $H^{*}\left(E_{\mathcal{S}}, \mathbb{Z}\right)$ under the $\operatorname{map} l_{\mathcal{S}}: c_{W} \mapsto\left[E_{W}\right]_{\mid E_{\mathcal{S}}}(W \neq 0)$ and $c_{0} \mapsto-\left[E_{0}\right]_{\mid E_{\mathcal{S}}}$.

Proof. For $V \in \mathcal{S} \cup\left\{\mathbb{C}^{n}\right\}$, let $p_{V}$ be the projection of $E_{\mathcal{S}}$ onto the factor $\mathbb{P}\left(\mathbb{C}_{V}^{\mathcal{S}}\right)^{\mathcal{G}_{V}^{\mathcal{S}}}$ associated to the decomposition in Proposition 4.5. Define a map of $\mathbb{Q}$-algebras

$$
p_{V}^{*}: \mathbb{Q}\left[\left[c_{W^{\prime}}\right]\right]_{W^{\prime} \in \mathcal{G}_{V}^{\mathcal{S}}} \longrightarrow \mathbb{Q}\left[\left[c_{W}\right]\right]_{W \in \mathcal{G}}
$$


as follows. For $W^{\prime} \neq 0$, let $c_{W^{\prime}} \mapsto c_{W}$, where $W=\pi^{-1}\left(W^{\prime}\right)$ and $\pi: V \rightarrow \mathbb{C}_{V}^{\mathcal{S}}$. For $W^{\prime}=0$, let

$$
c_{0} \mapsto \sum_{\substack{W \subset V_{\mathcal{S}}, W \in \mathcal{G} \\\{W\} \cup S \text { nested }}} c_{W}
$$

By Lemma 4.10, we have a commutative diagram of $\mathbb{Q}$-algebras

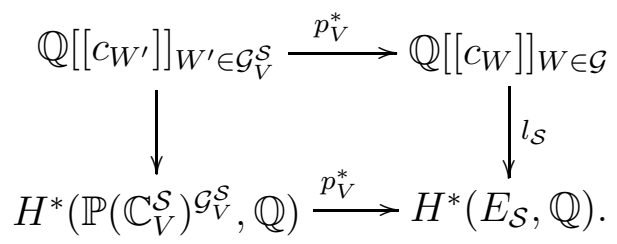

For $W^{\prime} \in \mathcal{G}_{V}^{\mathcal{S}}$, denote by $\bar{W}^{\prime}$ the subspace $\pi^{-1}\left(W^{\prime}\right)$ of $V$, where $\pi: V \rightarrow \mathbb{C}_{V}^{\mathcal{S}}$. Then $p_{V}^{*} G_{W^{\prime}}^{\mathcal{G}}=P_{W^{\prime}}^{\mathcal{S}, V}$. Then the Proposition follows from Lemma 4.6 and Corollary 4.9 .

Proof of Lemma 4.4. Let $\mathcal{E}$ be the invertible sheaf $\mathcal{O}_{E_{\mathcal{S}}}\left(\sum_{V \in \mathcal{G}} a_{V}^{\prime}(c) E_{V}\right)$. By definition, for $i \geq 0, c h_{i}(\mathcal{E})=(1 / i !)\left(\sum_{V \in \mathcal{G}} a_{V}^{\prime}(c)\left[E_{V}\right]_{\mid E_{\mathcal{S}}}\right)^{i}$. Theorem 2.3 allows us to write

$$
\chi\left(E_{\mathcal{S}}, \mathcal{E}\right)=\sum_{i+j=n-1-|\mathcal{S}|} \frac{1}{i !}\left(\sum_{V \in \mathcal{G}} a_{V}^{\prime}(c)\left[E_{V}\right]_{\mid E_{\mathcal{S}}}\right)^{i} \cdot T d_{j}\left(E_{\mathcal{S}}\right) .
$$

The Lemma follows from the map (3) and Proposition 4.11. Remark that the map $l_{\mathcal{S}}$ from Proposition 4.11, factors on homogenous polynomials of degree $n-1-|\mathcal{S}|$ via: multiplication of the map (3) with $\prod_{V \in \mathcal{S}} c_{V}$.

Proof of Theorem 1.4. By [B]- Proposition 2.7 (ii),

$$
n_{c, x}(D)=\chi\left(\widetilde{Y}, \mathcal{O}_{E^{\mathcal{S}_{c, x}}}\left(K_{\widetilde{Y} / Y}-\left\llcorner(c-\epsilon) \rho^{*} D\right\lrcorner\right)\right),
$$

for $0<\epsilon \ll 1$, where $\mathcal{S}_{c, x}$ is empty unless $\mathrm{cm}(D) \in \mathbb{Z}$ and there exists a divisor on $\tilde{Y}$ mapping onto $\{x\}$. In the later case, $\mathcal{S}_{c, x}=\left\{V_{x}\right\}$. Thus

$$
n_{c, x}(D)=\chi\left(\mathcal{O}_{E^{\mathcal{S}_{c, x}}}\left(\sum_{V \in \mathcal{G}-\{0\}} a_{V}(c) E_{V}\right)\right.
$$

and the Theorem follows by replacing $a_{0}$ with 0 in the proof of Lemma 4.4

\section{EXAMPLE}

The following easy example illustrates how Theorems 1.3 and 1.4 work. Let $D$ be the union of three distinct lines passing through one point in $\mathbb{P}^{2}$. Let $\mathcal{A}=$ $\left\{V_{1}, V_{2}, V_{3}\right\}, V_{i} \subset \mathbb{C}^{3}$ mutually distinct subspaces of dimension 2 , with $V_{1} \cap V_{2} \cap V_{3}=$ $L$ where $\delta(L)=1$. Then $D=\mathbb{P}\left(V_{1}\right)+\mathbb{P}\left(V_{2}\right)+\mathbb{P}\left(V_{3}\right)$ as a divisor in $\mathbb{P}\left(\mathbb{C}^{3}\right)=\mathbb{P}^{2}$. 
Take $\mathcal{G}=\left\{0, L, V_{1}, V_{2}, V_{3}\right\}$. By (2),$c_{0}+c_{L}+c_{V_{i}}(i=1,2,3)$ belongs to the ideal $I$. We can eliminate thus the variables $c_{V_{i}}(i=1,2,3)$ and have

$$
\mathbb{Q}\left[c_{V}\right]_{V \in \mathcal{G}} / I \cong \mathbb{Q}\left[c_{0}, c_{L}\right] /\left(c_{0}^{3}, c_{0} c_{L},\left(c_{0}+c_{L}\right)^{2}\right),
$$

and, by (3), this is isomorphic with the cohomology ring of $\tilde{Y}$, the blow up of $\mathbb{P}^{2}$ at $\mathbb{P}(L)$.

The only $c \in(0,1)$ for which $\mathcal{S}_{c} \neq \emptyset$ are $c=1 / 3,2 / 3$. For both cases, $\mathcal{S}_{c}=\{L\}$; call this set $\mathcal{S}$. We have

$$
T^{\mathcal{S}}=P_{0}^{\mathcal{S}, L} P_{L}^{\mathcal{S}, \mathbb{C}^{3}} \prod_{1 \leq i \leq 3} P_{V_{i}}^{\mathcal{S}, \mathbb{C}^{3}}
$$

From the fact that $Q(x)=1+\frac{1}{2} x+$ (degree $\geq 2$ terms), we get

$$
T^{\mathcal{S}}=1+\left(-\frac{3}{2} c_{0}-c_{L}\right)+(\text { degree } \geq 2 \text { terms })
$$

It follows by Theorem 1.3 that $c=1 / 3$ is a jumping number for $D$ if and only if $-\frac{5}{2} c_{0} c_{L}$ does not lie in the ideal $I \subset \mathbb{Q}\left[c_{V}\right]_{V \in \mathcal{G}}$. Also, $c=2 / 3$ is a jumping number if and only if $-\frac{5}{2} c_{0} c_{L}+c_{L}^{2}$ does not lie in $I$. Therefore $c=\frac{2}{3}$ is the only jumping number of $D$ in the interval $(0,1)$. By Theorem 1.4, the inner jumping multiplicity at $x=\mathbb{P}(L)$ of $c=2 / 3$ is given by writing $-\frac{3}{2} c_{0} c_{L}-c_{L}^{2} \in \mathbb{Q}\left[c_{V}\right]_{V \in \mathcal{G}} / I$ in terms of $c_{0}^{2}$. Thus $n_{x, \frac{2}{3}}(D)=1$. Also, $n_{x, 1}(D)$ is given by $-\frac{3}{2} c_{0} c_{L}-2 c_{L}^{2}$, hence $n_{x, 1}(D)=2$. This gives the initial part of the spectrum of $D$ at $x$, and in fact (in this case by symmetry) the whole spectrum is $t^{2 / 3}+2 t+t^{4 / 3}$.

\section{REFERENCES}

[B] N. Budur, On Hodge spectrum and multiplier ideals. Math. Ann. 327 (2003), no. 2, 257270.

[BS] N. Budur, and M. Saito, Multiplier ideals, $V$-filtration, and spectrum. J. Algebraic Geom. 14 (2005), no. 2, 269-282.

[DP] C. De Concini, and C. Procesi, Wonderful models of subspace arrangements. Selecta Math. (N.S.) 1 (1995), no. 3, 459-494.

[D] A. Dimca, Singularities and topology of hypersurfaces. Universitext. Springer-Verlag, New York, 1992. xvi+263 pp.

[F] W. Fulton, Intersection Theory. Second edition. Ergebnisse der Mathematik und ihrer Grenzgebiete. 3. Folge. A Series of Modern Surveys in Mathematics [Results in Mathematics and Related Areas. 3rd Series. A Series of Modern Surveys in Mathematics], 2. Springer-Verlag, Berlin, 1998. xiv+470 pp.

[L] R. Lazarsfeld, Positivity in algebraic geometry. II. Positivity for vector bundles, and multiplier ideals. Ergebnisse der Mathematik und ihrer Grenzgebiete. 3. Folge. A Series of Modern Surveys in Mathematics [Results in Mathematics and Related Areas. 3rd Series. A Series of Modern Surveys in Mathematics], 49. Springer-Verlag, Berlin, 2004. xviii +385 pp.

[M] M. Mustaţă, Multiplier ideals of hyperplane arrangements. Trans. Amer. Math. Soc. 358 (2006), no. 11, 5015-5023.

[S] M. Saito, Jumping coefficients and spectrum of a hyperplane arrangement. Preprint.

[St] J.H.M. Steenbrink, The spectrum of hypersurface singularities. Actes du Colloque de Théorie de Hodge (Luminy, 1987). Astérisque No. 179-180 (1989), 11, 163-184. 
[T] Z. Teitler, A note on Mustaţă's computation of multiplier ideals of hyperplane arrangements. Proc. Amer. Math. Soc. 136 (2008), 1575-1579.

Department of Mathematics, The University of Notre Dame, IN 46556, USA

E-mail address: nbudur@nd.edu 